\title{
Comparative genomic analysis of 1047 completely sequenced cDNAs from an Arabidopsis-related model halophyte, Thellungiella halophila
}

Teruaki Taji ${ }^{*}$, Kenji Komatsu ${ }^{1,2}$, Taku Katori ${ }^{1}$, Yoshikazu Kawasaki ${ }^{1}$, Yoichi Sakata', Shigeo Tanaka', Masatomo Kobayashi ${ }^{3}$, Atsushi Toyoda ${ }^{4,5}$, Motoaki Seki $^{6}$, Kazuo Shinozaki $^{6}$

\begin{abstract}
Background: Thellungiella halophila (also known as T. salsuginea) is a model halophyte with a small size, short life cycle, and small genome. Thellungiella genes exhibit a high degree of sequence identity with Arabidopsis genes ( $90 \%$ at the cDNA level). We previously generated a full-length enriched CDNA library of T. halophila from various tissues and from whole plants treated with salinity, chilling, freezing stress, or ABA. We determined the DNA sequences of 20000 cDNAs at both the 5'- and 3' ends, and identified 9569 distinct genes.

Results: Here, we completely sequenced 1047 Thellungiella full-length cDNAs representing abiotic-stress-related genes, transcription factor genes, and protein phosphatase $2 \mathrm{C}$ genes. The predicted coding sequences, 5'-UTRs, and $3^{\prime}$-UTRs were compared with those of orthologous genes from Arabidopsis for length, sequence similarity, and structure. The 5'-UTR sequences of Thellungiella and Arabidopsis orthologs shared a significant level of similarity, although the motifs were rearranged. While examining the stress-related Thellungiella coding sequences, we found a short splicing variant of T. halophila salt overly sensitive 1 (ThSOS1), designated ThSOS1S. ThSOS1S contains the transmembrane domain of ThSOS1 but lacks the C-terminal hydrophilic region. The expression level of ThSOS1S under normal growth conditions was higher than that of ThSOS1. We also compared the expression levels of Na ${ }^{+}$-transport-system genes between Thellungiella and Arabidopsis by using full-length cDNAs from each species as probes. Several genes that play essential roles in $\mathrm{Na}^{+}$excretion, compartmentation, and diffusion (SOS1, SOS2, NHX1, and HKT1) were expressed at higher levels in Thellungiella than in Arabidopsis.
\end{abstract}

Conclusions: The full-length cDNA sequences obtained in this study will be essential for the ongoing annotation of the Thellungiella genome, especially for further improvement of gene prediction. Moreover, they will enable us to find splicing variants such as ThSOS1S (AB562331).

\section{Background}

Thellungiella halophila (also known as T. salsuginea) is used as a model system for understanding abiotic stress tolerance. It shows tolerance not only to extreme salinity stress, but also to chilling, freezing, and ozone stresses [1-10]. Thellungiella is closely related to Arabidopsis, with 90\% cDNA sequence identity between the two species, and it can be easily transformed by using the floral dipping

\footnotetext{
* Correspondence: t3teruak@nodai.ac.jp

'Faculty of Applied Bioscience, Tokyo University of Agriculture, 1-1-1

Sakuragaoka, Setagaya-ku, Tokyo 156-8502, Japan

Full list of author information is available at the end of the article
}

method $[1,11]$. Thellungiella has a number of other features useful for genetic research, such as small size, short life cycle, high seed number, and self-compatibility.

The Arabidopsis genome sequence and other genetic resources, including collections of full-length cDNAs, have provided powerful tools for comparative genomics to understand the biology and evolution of other plants $[3,5,12]$. In particular, highly accurate full-length cDNA sequences that span the entire protein-coding region of a given gene can advance comparative, functional, and structural genome analyses. The accurate prediction of protein-coding regions in genome sequences is limited 
by the difficulty of finding islands of coding sequences within an ocean of noncoding DNA, and by the complexity of individual genes that may code for multiple peptides through alternative splicing. The sequence data from full-length cDNAs has contributed to the accuracy of annotation and to improving gene prediction in Arabidopsis [13-15]. For these reasons, we have been working to collect similar data for Thellungiella.

We previously reported construction of a full-length cDNA library of Thellungiella derived from various tissues and from whole seedlings subjected to environmental stress treatments, including high salinity, chilling, freezing, and abscisic acid (ABA). We obtained a total of 35171 sequences from 20000 clones, and named them RIKEN Thellungiella Full-length (RTFL) cDNA clones. These sequences were assembled by using the CAP3 method and were clustered into 9569 nonredundant cDNA groups [16].

Thellungiella has an effective system for suppressing $\mathrm{Na}^{+}$influx and for excreting $\mathrm{Na}^{+}$[3]. It also exhibits high potassium/sodium selectivity, according to electrophysiological analysis of instantaneous current [4]. This implies that Thellungiella has ion channels with specific features that lead to superior sodium/potassium homeostasis. Membrane transporters have been shown to be important components of salt tolerance mechanisms in other species on account of their regulation of ion homeostasis. For example, the SALT OVERLY SENSITIVE (SOS) pathway is a well-defined pathway in Arabidopsis for the regulation of sodium ion homeostasis during plant growth under salinity stress $[17,18]$. In this pathway, a calcium-binding protein, SOS3, perceives a change in intracellular calcium concentration induced by salt stress and then binds to and activates SOS2, a serine/threonine protein kinase. The SOS3-SOS2 complex increases the expression and activity of SOS1, which encodes a plasma membrane $\mathrm{Na}^{+} / \mathrm{H}^{+}$exchanger (antiporter) $[19,20]$. Activated SOS1 transports cytosolic sodium out of the cell, reducing the cellular build-up of toxic levels of sodium [17]. The Thellungiella SOS1 gene, ThSOS1, has a conserved amino acid sequence and protein structure with orthologous genes from Arabidopsis and other plants [21]. Transgenic Thellungiella plants in which ThSOS1 transcript levels were reduced by RNA interference (RNAi) showed lower salt tolerance than wild-type plants, suggesting that SOS1 is critical for salt tolerance in halophytic species as well as in glycophytic species such as Arabidopsis [21]. Recently, a 193-kb Thellungiella BAC clone containing the putative SOS1 locus was sequenced, annotated, and compared with the sequence in the orthologous $146-\mathrm{kb}$ region of the Arabidopsis genome on chromosome 2 [22].

Here, we selected 1047 cDNAs for genes related to salt stress, transcription factors, transporters, and protein phosphatase 2Cs from 9569 individual RTFL clones, and determined the complete sequences. We then predicted the coding sequence (CDS), $5^{\prime}$-UTR, and 3'-UTR for each of the cDNAs and compared them with the corresponding regions from the orthologous Arabidopsis genes. We also compared the expression levels of Thellungiella and Arabidopsis $\mathrm{Na}^{+}$-transport system genes by using full-length cDNAs to probe Northern blots under equal conditions of hybridization and detection.

\section{Results and Discussion}

\section{Selection and complete sequencing of 1047 full-length cDNAs}

We selected 1047 cDNA clones representing salt-stressrelated genes, transcription factors, and protein phosphatase 2Cs for full-insert sequencing (Table 1). The 1047 cDNA clones were completely sequenced, and the CDS, 5'-UTR, and 3'-UTR were predicted for each (see Additional file 1, Table S1 and Additional file 2, Table S2). The distribution of 5'-UTR, CDS, and 3'-UTR lengths is illustrated in Figure 1; their average lengths were $206 \pm 295 \mathrm{bp}, 1214 \pm 650 \mathrm{bp}$, and $270 \pm 283 \mathrm{bp}$, respectively. These results are comparable to the results obtained for CAP-trapper full-length cDNA collections from other plant species, including Arabidopsis (5'-UTR, 149 bp; CDS, 1206 bp; 3'-UTR, 238 bp; TAIR8 dataset of The Arabidopsis Information Resource (TAIR, ftp:// ftp.arabidopsis.org/home/tair/Sequences/blast_datasets/ TAIR8_blastsets/), maize (5'-UTR, 99 bp; 3'-UTR, 206 bp [23]), rice (5'-UTR, 259 bp; 3'-UTR, 398 bp [24]), and poplar (5'-UTR, 109 bp; 3'-UTR, 228 bp [25]).

\section{Comparison of CDS, 5'-UTR, and 3'-UTR sequences between orthologous genes in Thellungiella and Arabidopsis}

To assess the quality of the completely sequenced cDNAs, we performed BLAST analysis using CDS sequences against nucleotide or peptide sequences from the TAIR8 dataset (see Additional file 1, Table S1 and Additional file 2, Table S2) and identified Arabidopsis orthologs of the 1047 Thellungiella genes. The average lengths of the Arabidopsis orthologous CDSs, 5'-UTRs, and 3'-UTRs were $1331 \pm 698 \mathrm{bp}, 160 \pm 145 \mathrm{bp}$, and $241 \pm 146 \mathrm{bp}$, respectively. Figure 2 compares lengths and identities between the CDS, 5'-UTR, and 3'-UTR regions of the 1047 Thellungiella cDNAs and those of the orthologous genes from Arabidopsis. Most CDS pairs showed highly similar lengths, whereas the $5^{\prime}$ - and 3'-UTR pairs showed significant variation in length (Figure $2 \mathrm{~A}$ ). The average nucleotide identity within homologous CDS pairs was $87 \%$, whereas the average identity within the 5'- and 3'-UTR pairs was only $57 \%$ to $61 \%$ (Figure 2B). A previous analysis of the transcriptional 
Table 1 Classification of 1250 full-sequenced cDNAs

\begin{tabular}{|c|c|c|}
\hline classification & number of cDNAs & references \\
\hline 433 salt stress related genes & 367 & \\
\hline Thellungiella salt stress inducible genes & 127 & $\begin{array}{l}\text { Taji et al., } 2004 \text { Plant Physiol } \\
\text { Gong et al., } 2005 \text { Plant J. } \\
\text { Wong et al., } 2005 \text { Plant Mol Biol. } \\
\text { Taji et al., unpublished data }\end{array}$ \\
\hline salt stress inducible genes in Thellungiella and Arabidopsis & 191 & \\
\hline constitutive high-expressed genes in Thellungiella compared with Arabidopsis & 78 & \\
\hline genes encoding $\mathrm{Na}^{+}$transporter & 9 & \\
\hline overlapping genes in EST libraries of abiotic stressed plants from Thellungiella & 30 & \\
\hline response to abiotic or biotic stress classified by Gene Ontology ${ }^{a}$ & 450 & Taji et al., 2009 BMC Plant Biol. \\
\hline transcription factor classified by Gene Ontology ${ }^{a}$ & 285 & Taji et al., 2009 BMC Plant Biol. \\
\hline protein phosphatase $2 \mathrm{C}$ & 24 & Taji et al., 2009 BMC Plant Biol. \\
\hline total & 1126 & \\
\hline total after elimination of overlapped cDNAs & 1047 & \\
\hline
\end{tabular}

${ }^{a}$ Gene Ontlogy (GO) terms were obtained using InterPro. According to the GO terms, RTFL clones were classified by using Plant GO Slim http://www. geneontology.org/GO.slims.shtml

differences between Thellungiella and Arabidopsis showed that Arabidopsis has a global defense strategy that requires bulk gene expression, while Thellungiella induces expression of genes functioning in protein folding, posttranslational modification, and protein redistribution [5]. The sequence diversity in the 5'- and 3'-UTR pairs may be involved in the posttranslational regulation of stress tolerance mechanisms in Thellungiella.

\section{Comparison of structure of UTR regions between}

\section{Thellungiella and Arabidopsis}

To compare the overall architecture of the UTRs between Thellungiella and Arabidopsis, we randomly selected 10 orthologous pairs with 5'-UTRs of least $50 \mathrm{bp}$ in length from PP2Cs, transcription factors and transporters, respectively. We identified motif families shared between the 5'-UTRs of Thellungiella and Arabidopsis orthologs using the Dragon Motif Builder system [26]. Analyzing each of the orthologous pairs individually, we compared the order of the shared motifs between each pair (Additional file 3, Figure S1). Figure 3 shows the arrangement of the motifs in 5'-UTR regions in nine orthologous gene pairs in Thellungiella and Arabidopsis. These motif sequences are shown in Additional file 4, Table S3. The members of each orthologous 5'-UTR pair shared 4 to 19 motifs; however, positional rearrangements were found between the members of each pair. Similar positional rearrangements of 5'-UTR motifs were

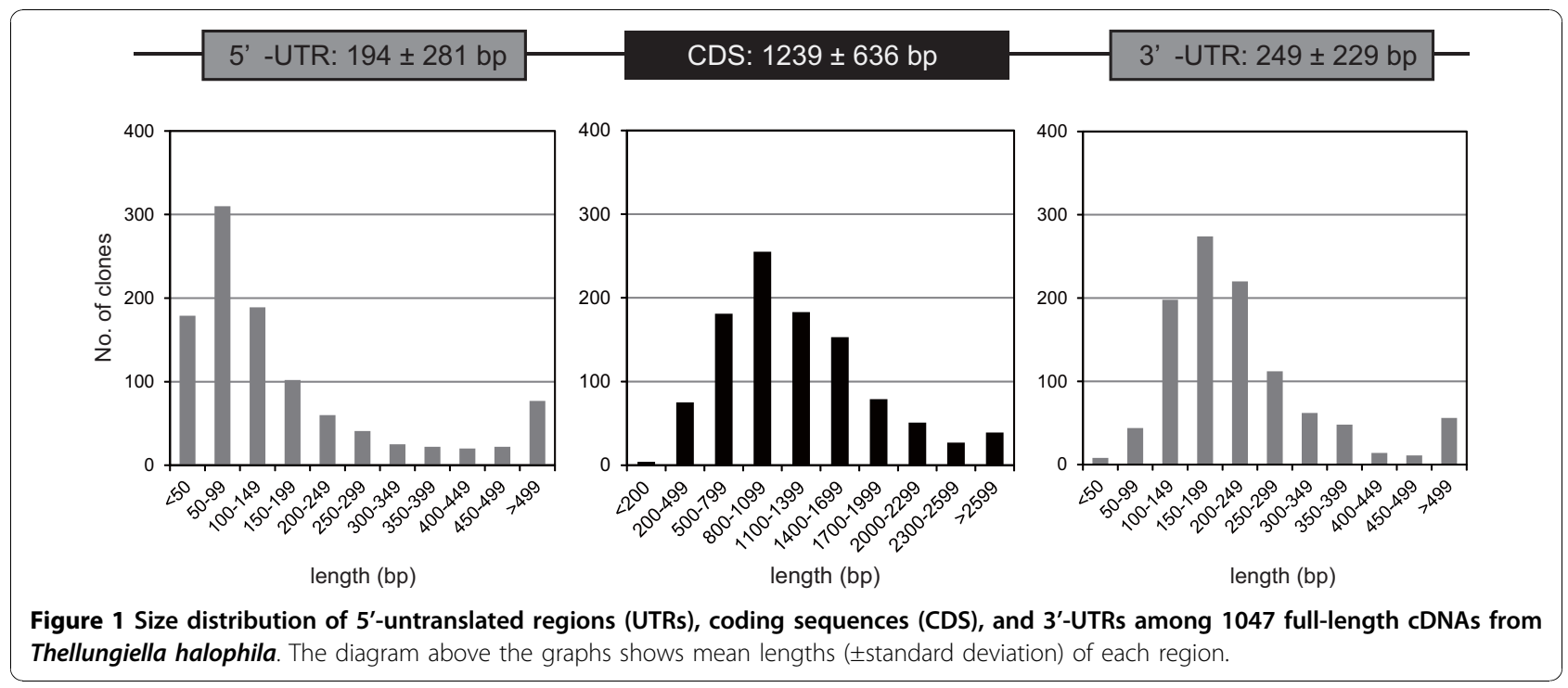




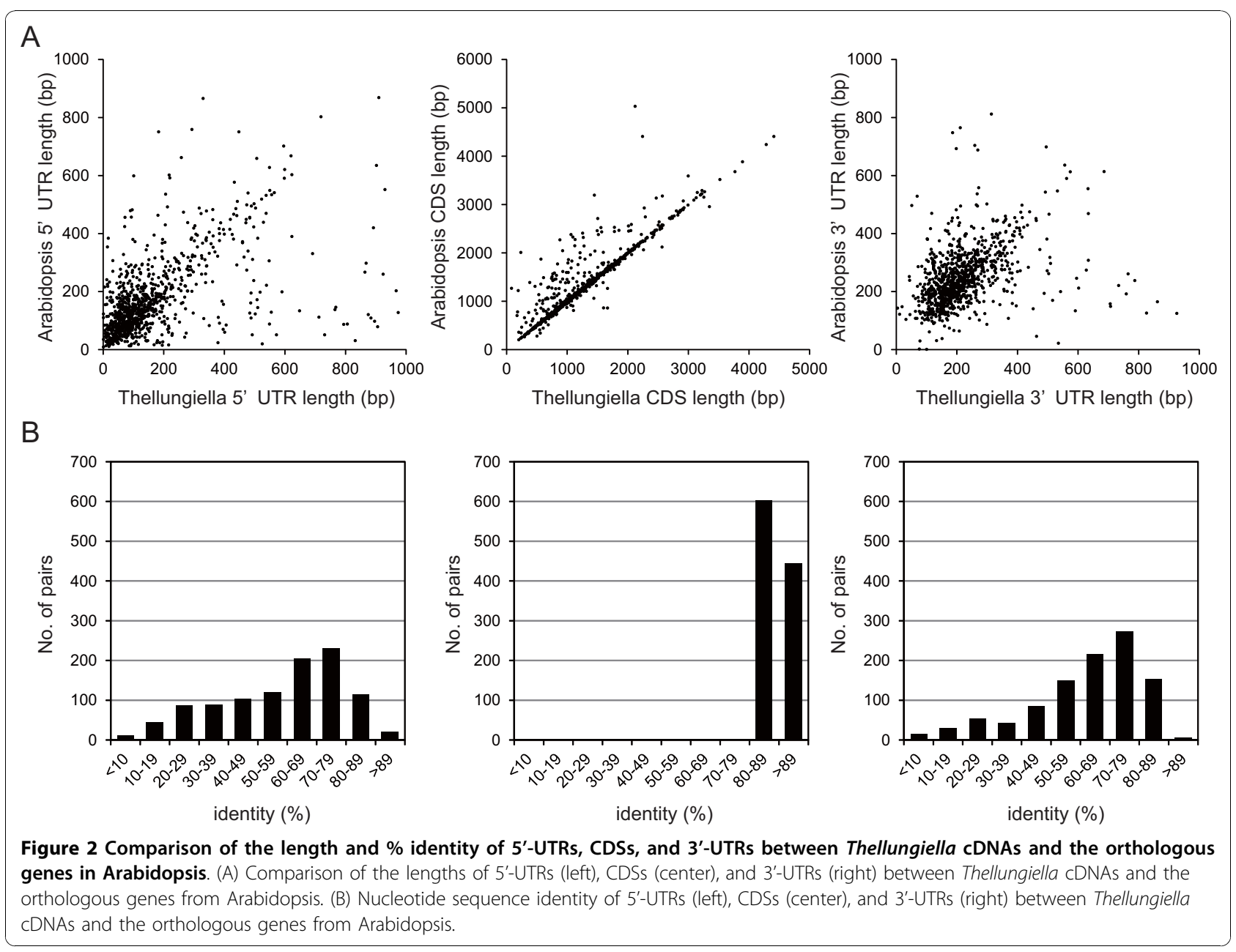

reported in a comparison of 48 pairs of orthologous sequences between common carp and zebrafish [27]. The presence of such shared motif families suggests the existence of regulatory components common to both species.

\section{Structural comparison of ThSOS1 and splicing variant ThSos1s}

Only one clone was orthologous to Arabidopsis SOS1 among 20000 sequenced Thellungiella cDNAs [16]. The deduced protein was a splice variant of ThSOS1 (Acc. No. EF207775.1). In the variant, an exon encoding 19 amino acid (aa) residues (60 nucleotides) followed by a stop codon was inserted at the beginning of the 15th exon of ThSOS1 (Figure 4A, B). We named the short variant ThSOS1S, for Thellungiella halophila Salt Overly Sensitive 1 Short form. ThSOS1 comprises an N-terminal, integral membrane domain (responsible for $\mathrm{Na}^{+}$transport) and a C-terminal hydrophilic region. In contrast, the predicted ThSOS1S protein has the transmembrane domain of ThSOS1 but lacks the C-terminal hydrophilic region, because the stop codon occurs just after the sequence encoding the transmembrane domain (Figure 4C).
The transmembrane portion of ThSOS1/ThSOS1S has sequence similarities with plasma membrane $\mathrm{Na}^{+} / \mathrm{H}^{+}$ exchangers of animal, bacterial, and fungal cells [20]. In animal cells, $\mathrm{Na}^{+} / \mathrm{H}^{+}$exchanger 1 (NHE1) functions as a $\mathrm{Na}^{+} / \mathrm{H}^{+}$antiporter to maintain $\mathrm{pH}$ homeostasis [28]. NHE1 has a C-terminal tail of $\sim 300$ aa, which is important in regulating the $\mathrm{Na}^{+} / \mathrm{H}^{+}$antiporter activity through phosphorylation or binding of regulatory proteins [29]. The Synechocystis $\mathrm{Na}^{+} / \mathrm{H}^{+}$antiporter SynNhaP also has a long hydrophilic C-terminal tail (100 aa). In SynNhaP, the deletion of a 56-aa hydrophilic terminal region partially inhibited the antiporter activity, and replacement of the long $\mathrm{C}$-terminal tail with the orthologous region from the halotolerant cyanobacterium Aphanothece halophytica, ApNhaP, altered its ion specificity [30]. Arabidopsis $\mathrm{Na}^{+} / \mathrm{H}^{+}$antiporter SOS1 has 12 predicted transmembrane domains in the $\mathrm{N}$-terminal region and a long cytoplasmic tail of $\sim 700$ aa at the C-terminus [20]. The predicted cytoplasmic tail of SOS1 interacts with radical-induced cell death 1 (RCD1), a regulator of oxidative stress responses under salt or oxidative stress. Like rcd1 mutants, sos 1 mutants show an altered 


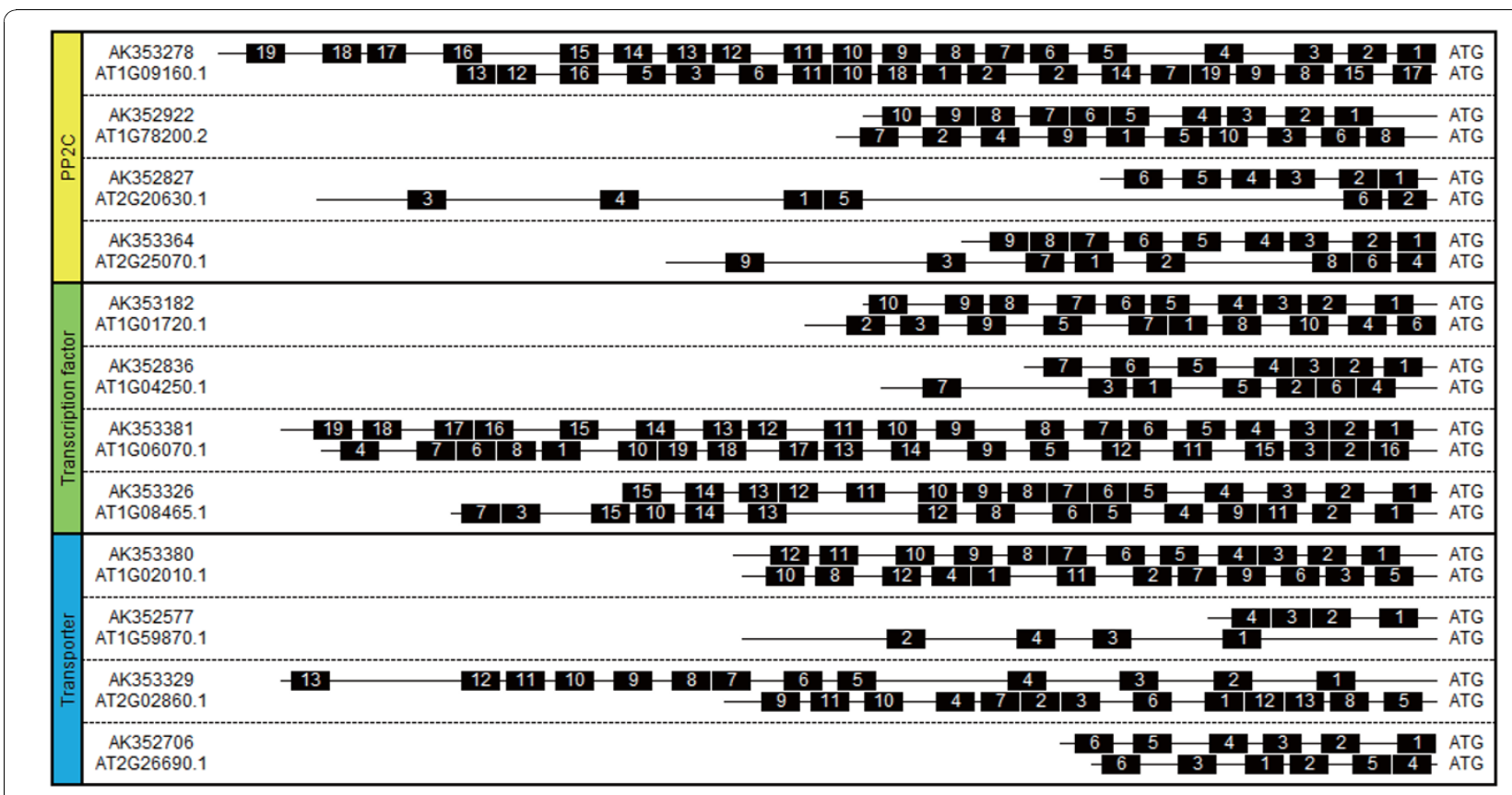

Figure 3 Arrangement of motifs identified in 5'-UTR regions of nine orthologous gene pairs in Thellungiella and Arabidopsis. The motif families shared in 5'-UTRs of nine randomly selected Thellungiella and Arabidopsis orthologous pairs from PP2Cs, transcription factors and transporters were identified using Dragon Motif Builder [26]. Black boxes indicate motifs. Identical numbers within each pair indicate identical motifs. Motif sequences are shown in Additional file 4, Table S3.

sensitivity to oxidative stresses [31]. These results suggest that the long C-terminal tail mediates not only the regulation of transport activity with a variety of intracellular regulatory proteins, but also the ion specificity and the cross-talk with other stress tolerance mechanisms.

The N-terminal transmembrane region of SOS1 shows high similarity among various organisms (Figure 4A), whereas there is no significant similarity among the Cterminal regions [30]. The $\mathrm{C}$-terminal sequence variation may result in different functions for this region among different organisms. In particular, NhaP, a Na ${ }^{+} / \mathrm{H}^{+}$antiporter of Pseudomonas aeruginosa, is highly homologous to SOS1, NHE1, SynNhaP, and ApNha1 (Figure 4A), but it does not have the C-terminal long tail [32]. ThSOS1S is similar to NhaP in that it contains only a $\mathrm{Na}^{+} / \mathrm{H}^{+}$-exchanger domain in the transmembrane domain. It is possible that ThSOS1S functions as an $\mathrm{Na}$ ${ }^{+} / \mathrm{H}^{+}$antiporter whereas ThSOS1 functions not only in salt stress response (via the $\mathrm{N}$-terminal $\mathrm{Na}^{+} / \mathrm{H}^{+}$antiporter), but also in response to other abiotic stresses (via the long C-terminal tail).

\section{Expression levels of ThSOS1 and ThSOS1S}

We performed qRT-PCR analysis of ThSOS1 and ThSOS1S expression by using primers specific to each of these splice variants (Figure 5A). We detected both transcripts, suggesting that Thellungiella normally produces both forms (Figure 5B). Interestingly, the expression level of ThSOS1S under normal growth conditions was higher than that of ThSOS1. The expression level of SOS1 in Thellungiella is higher than that in Arabidopsis when full-length cDNAs are used as probes, especially under normal growth conditions [3]. These data suggest that the high expression of ThSOS1 detected under normal growth conditions derive from the high expression level of ThSOS1S. To confirm the existence of such a similar splice variant in Arabidopsis, RT-PCR was performed using primer sets that are able to detect the splice variants in Thellungiella and Arabidopsis. The short splice variant corresponding to ThSOS1S was not detected in Arabidopsis, whereas both splice variants were detected in Thellungiella (Figure 5C). This result suggests that the short splice variant of SOS1 is specific to Thellungiella.

\section{Expression profiles of $\mathrm{Na}^{+}$transport genes of}

Thellungiella and Arabidopsis

The set of completely sequenced Thellungiella cDNA clones contains several genes that function in the $\mathrm{Na}^{+}$ transport system, including SOS1, NHX1, NHX2, NHX5, and high affinity $\mathrm{K}^{+}$transporter 1 (HKT1). We performed RNA blot analysis of these genes in both Thellungiella and Arabidopsis using full-length cDNAs as probes. In each case, RNA blots of a given species were hybridized with probes derived from that same species, with conditions of probe radioactivity, hybridization, and 
A
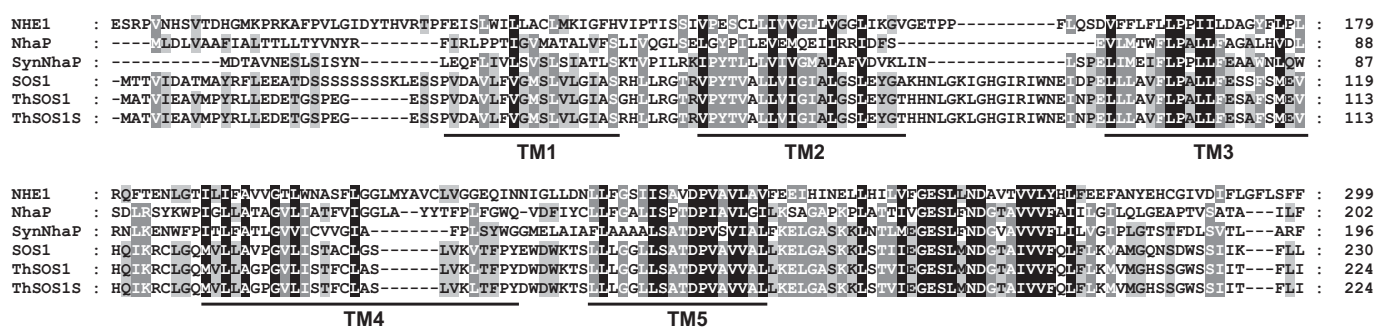

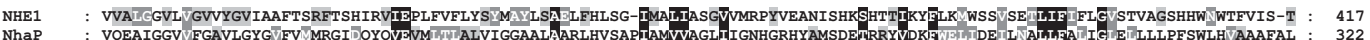

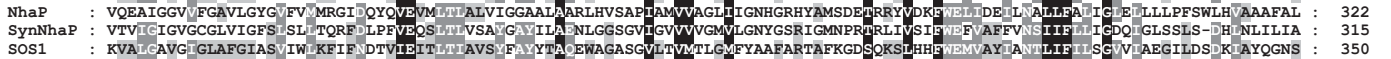

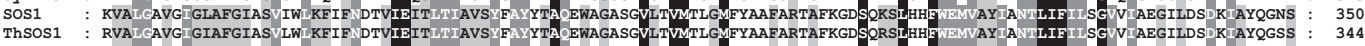

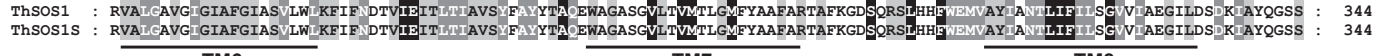

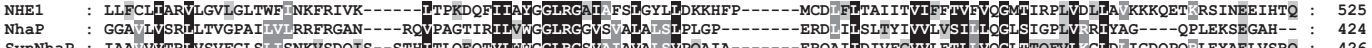

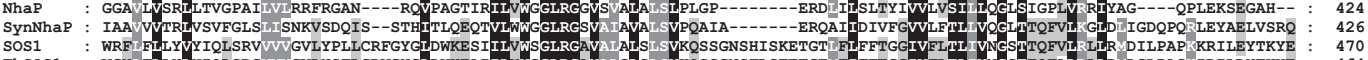

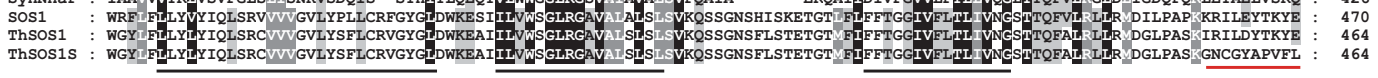

NHE1 : FLDHLITGIEDICGHYGHHHWDKLNRFNKKYVKKCLIAGERSKEPQLIAFYHKMEMKQAIELVESGGMGKIPSAVSTVSMQNIHPKSLPSERILPALSKDNEEEIRKILRNNLQKTRQR : 645

SYnNhaP : IALRRVLAELEKTDEF--PDINPERLRYKQELVQG-QLQSVTDKLKLLLLEYPLIQEVANKKFDQTVLDIEAETYADLIRMGRLEENIMPLLVTLEGENVAEPS-----SOS1 : MLNKALRAFQDLGDDE--ELGPADWPTVESYISSLKGSEGELVHHPHNGSKIGSLDPKSLKDIRMRFLNGVQATYWEMLDEGRISEVTANILMQSVDEALDQVSTT-LCDWRGLKPHVNF : 587 ThSOS1 : MLNKALQAFEDLGDE--ELGPADWPTVESYISSLKDSEGEQVHP-HSGSKPGNLDHTSLKDIRIRFLNGVQAAYWEMLDEGRISESTANILMRSVDEALDHISTEPLCDWRGLKSHVKF : 58

B

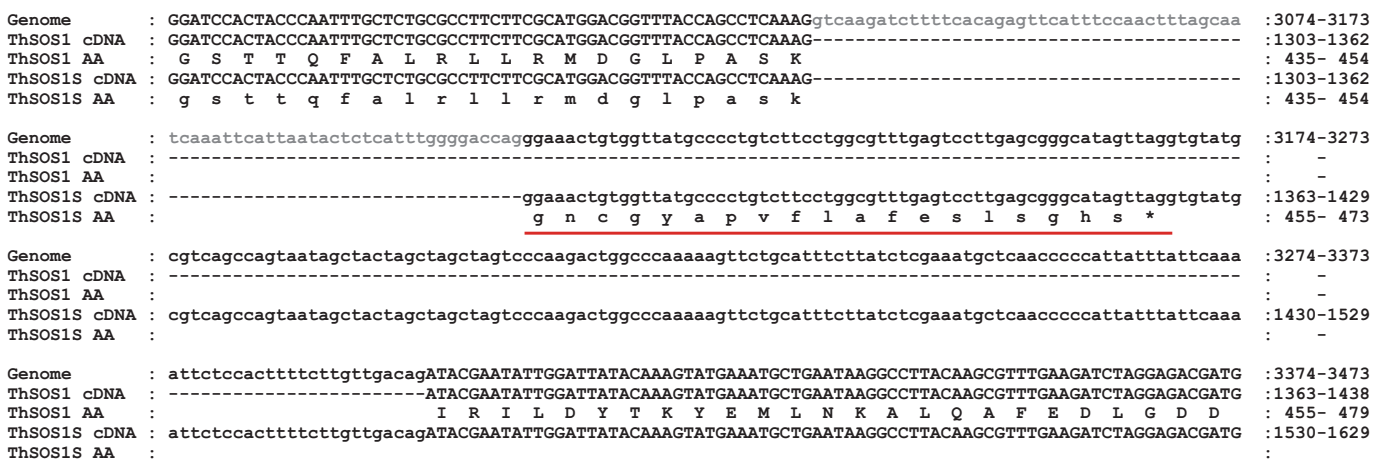

C

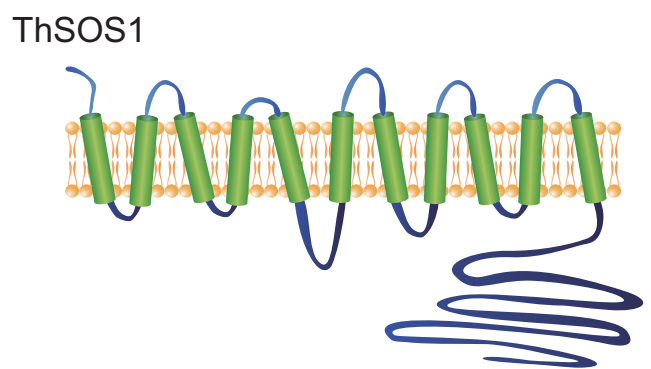

ThSOS1S

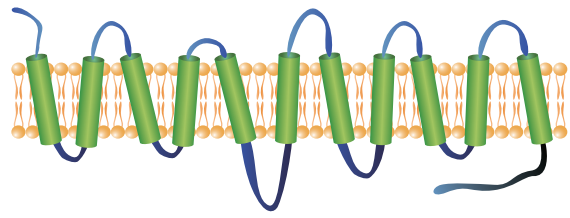

Figure 4 Comparison of the structures of ThSOS1 and ThSOS1S. (A) Alignment of the transmembrane regions of NHE1 (P19634), SynNhaP (D90910), SOS1 (AF256224), and ThSOS1 (EF207775.1) and the complete sequences of NhaP (P13738) and ThSOS1S constructed with ClustalW ver. 1.83. Amino acid residues conserved in all sequences are highlighted in black, and conserved substitutions are shown in gray. Predicted membrane spanning regions (TM) were determined by TMpred [42] and are marked under the alignment. (B) Close-up alignment of the nucleotide sequences of ThSOS1, ThSOS1S, and the genome with the deduced amino acid sequences from Gly-435 to Asp-479. The nucleotides conserved in ThSOS1 and ThSOS1S are written in capital letters, and the ThSOS1S-specific sequence is written in lower-case letters. Intron sequence is shown in gray letters. The predicted amino acid sequence of ThSOS1 is shown in capital letters, and that of ThSOS1S in lower-case letters. The 19aa insert of ThSOS1S is red-underlined. The asterisk shows the point at which the ThSOS1S protein is truncated. (C) Hypothetical secondary structure models of ThSOS1 and ThSOS1S. 

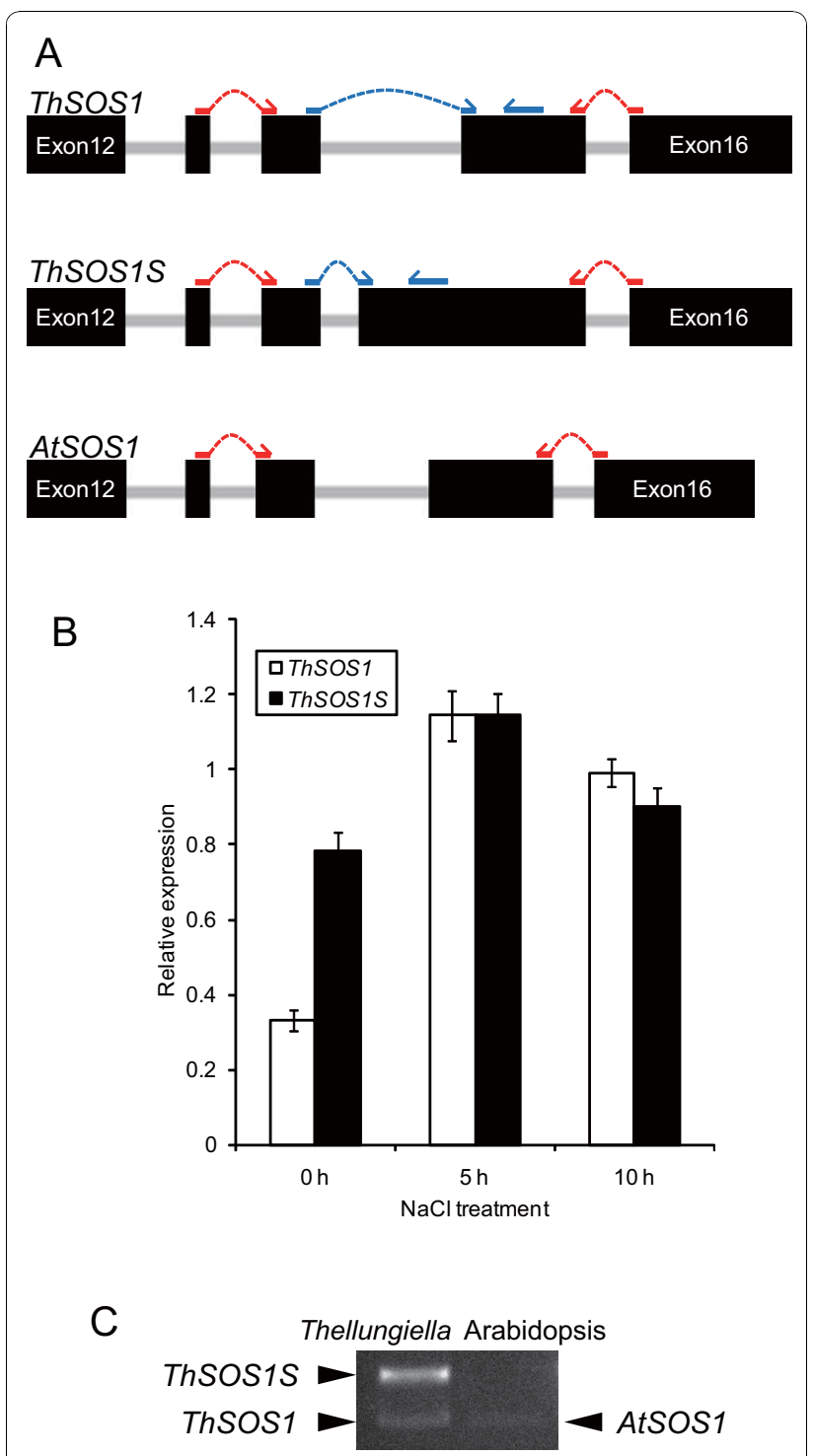

Figure 5 Effect of salt stress on transcript levels of ThSOS1 and ThSOS1S. (A) Schematic of the corresponding regions of the primers in ThSOS1, ThSOS1S and AtSOS1 for RT-PCR analysis. Exons are indicated as filled boxes and introns as spaces between them. (B) Relative expression of ThSOS1 and ThSOS1S under salt stress. Two-week-old Thellungiella seedlings were exposed to $250 \mathrm{mM} \mathrm{NaCl}$ stress for 0 , 5, or $10 \mathrm{~h}$. Total RNA was reversed-transcribed into CDNA and used as a template for quantitative RT-PCR. The qRT-PCR performed using blue-primer sets in (A). Relative transcript levels were normalized to Actin2 mRNA. Data are the mean \pm SD for three individual experiments $(n=3)$. (C) Semiquantitative gene expression of SOS1 splice variants in Thellungiella and Arabidopsis under normal growth conditions. The RT-PCR performed using red-primer sets in (A), which were able to detect these splice variants.

exposure period normalized between the two species. The expression levels of SOS1, NHX1, NHX2 and HKT1 in Thellungiella were higher than those in Arabidopsis under both normal and high-salinity conditions (Figure 6). SOS1, NHX1, and HKT1 play essential roles in salt tolerance in Arabidopsis [33-35], and transgenic plants overexpressing either SOS1 or NHX1 show higher tolerance to salt stress than do wild-type plants $[18,36]$. In particular, the expression level of NHX1 was very high in Thellungiella under both normal- and high-salinity conditions, suggesting that the constitutively high expression of molecules functioning in $\mathrm{Na}^{+}$transport may partly account for the high salinity tolerance of Thellungiella.

It is difficult to understand the relationship between $H K T 1$ expression and salinity tolerance. On the one hand, the overexpression of AtHKT1 increased sensitivity to $\mathrm{NaCl}$ (but not to $\mathrm{LiCl}$ or $\mathrm{KCl}$ ) compared with wild-type plants [37]. On the other hand, the ectopic production of wheat HKT1 containing specific amino acid substitutions (A240V, Q270L, or N365S) enhanced $\mathrm{NaCl}$ tolerance in yeast [38]. We compared the deduced amino acid sequences of HKT1 among Arabidopsis, Thellungiella, and wheat to search for point mutations inducing higher salt tolerance in Thellungiella HKT1. However, none of the mutations that gave high salt tolerance in the wheat HKT1 experiment was found in Thellungiella HKT1 (data not shown). It remains unknown whether other differences in the HKT1 sequences between Thellungiella and Arabidopsis confer salinity tolerance to Thellungiella. Arabidopsis HKT1 transports only $\mathrm{Na}^{+}$, not $\mathrm{K}^{+}$, because in the $\mathrm{K}^{+}$channel motif GYG, which is critical for $\mathrm{K}^{+}$selectivity, the first glycine is replaced with serine (Ser-68) [39]. The same position in the Thellungiella HKT1 ortholog is also

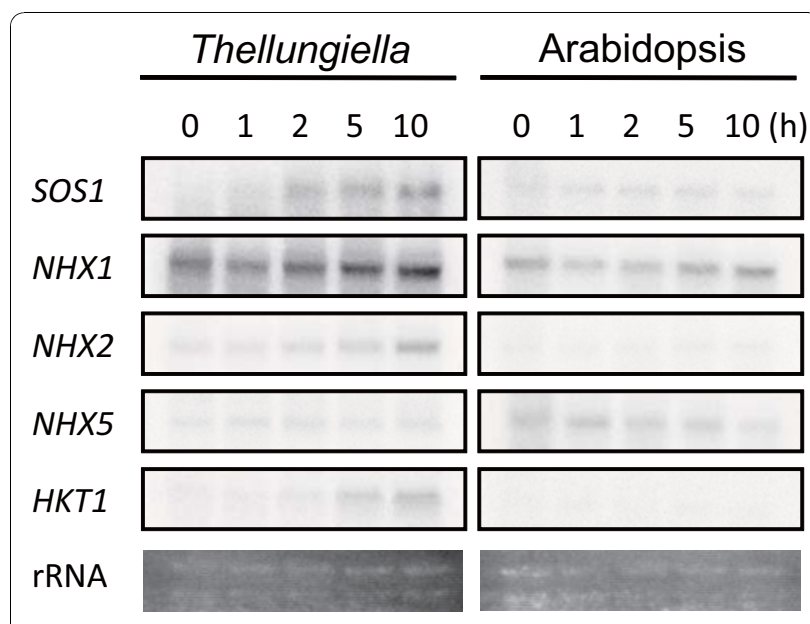

Figure 6 RNA gel blot analysis of $\mathrm{Na}^{+}$transport system genes in Thellungiella and Arabidopsis. Total RNA was prepared from 3week-old Thellungiella and Arabidopsis plants subjected to $250 \mathrm{mM}$ $\mathrm{NaCl}$ stress treatment for the periods indicated. Each lane was loaded with $10 \mu \mathrm{g}$ total RNA. The probes were the following fulllength cDNA fragments from Thellungiella and Arabidopsis, respectively: SOS1 (AK353556), NHX1 (AK352788), NHX2 (AK353509), NHX5 (AK353024), and HKT1 (AK353477). 
serine (Ser-68), suggesting that Thellungiella HKT1 also transports only $\mathrm{Na}^{+}$.

\section{Conclusions}

We sequenced 1047 Thellungiella cDNAs and used this information to compare the responses of Thellungiella and Arabidopsis to high-salinity conditions. The fulllength cDNA sequences will contribute to annotation of the Thellungiella genome and will improve gene predictions. Moreover, these fully sequenced cDNAs will enable finding splicing variants such as ThSOS1S. RNA blot analysis indicated that the extreme salt tolerance of Thellungiella might be attributable to the constitutively higher expression of genes functioning in the $\mathrm{Na}^{+}$transport system.

\section{Data access}

Sequences from this study have been deposited in NCBI GenBank under accession numbers [GenBank: AK352512] to [GenBank: AK353558]. The RTFL clones are available for distribution from the RIKEN Bioresource Center http://www.brc.riken.go.jp/lab/epd/Eng/.

\section{Methods}

Determination of CDSs, 5'-UTRs, and 3'-UTRs of full-length cDNAs

The locations of CDSs were determined with the EMBOSS getorf program (ver. 6 [40]), which identifies the longest stretch of uninterrupted sequence between a start codon (ATG) and stop codon (TGA, TAG, TAA) in the 5'- to 3' direction as the predicted CDS. The sequences before and after each predicted CDS were designated as the 5'- and 3'-UTRs, respectively. The 3' poly(A)-tail lengths were not included when determining the UTR lengths.

\section{Identification of orthologous genes in Arabidopsis and Thellungiella}

The CDS data set of 1047 Thellungiella cDNAs was compared with the gene sequences in The Arabidopsis Information Resource (TAIR8) by using BLAST searches (ver. 2.2.17 [41]). The top hit in each BLAST search was assumed to be the Arabidopsis ortholog.

\section{Plant materials and growth conditions}

Seeds of Thellungiella halophila (Shang Dong ecotype) and Arabidopsis thaliana (Columbia-0 ecotype) were sown on MS agar plates containing full-strength MS, $0.8 \%(\mathrm{w} / \mathrm{v})$ agar, and $1 \%$ sucrose with vitamin mixture (10 mg L $\mathrm{m}^{-1}$ myoinositol, $200 \mu \mathrm{g} \mathrm{L}^{-1}$ glycine, $50 \mu \mathrm{g} \mathrm{L}^{-1}$ nicotinic acid, $50 \mu \mathrm{g} \mathrm{L}^{-1}$ pyridoxine hydrochloride, $10 \mu \mathrm{g}$ $\mathrm{L}^{-1}$ thiamin hydrochloride, $\mathrm{pH}$ 5.7) and the plates were sealed with surgical tape. The seeds were stratified at $4^{\circ} \mathrm{C}$ for 7 days and then transferred to $80 \mu \mathrm{mol} \mathrm{m} \mathrm{m}^{-2}$ irradiance under an $8 / 16$-h day/night cycle at $22^{\circ} \mathrm{C}$ for germination and growth.

\section{Northern analysis}

Three-week-old Arabidopsis and Thellungiella plants that had been grown on $1 / 2 \mathrm{MS}$ plates were soaked in $250 \mathrm{mM} \mathrm{NaCl}$ solution for 1, 2, 5, or $10 \mathrm{~h}$. Total RNA was extracted by using RNAiso reagent (TaKaRa, Japan). Total RNA $(10 \mu \mathrm{g})$ was fractionated in $1 \%$ agarose gel containing formaldehyde and blotted onto a nylon membrane using 20× SSC. DNA fragments of the fulllength cNDAs for AtSOS1 (RAFL09-06-M16), AtSOS2 (RAFL09-61-G03), AtNHX1 (RAFL09-87-C17), AtNHX2 (RAFL07-95-G07), AtNHX5 (RAFL09-15-L23), AtHKT1 (RAFL15-31-F16), ThSOS1 (RTFL01-052_J19), ThSOS2 (RTFL01-012_C03), ThNHX1 (RTFL01-029_F03), ThNHX2 (RTFL01-047_K14), ThNHX5 (RTFL01046_D22) and ThHKT1 (RTFL01-044_N15) were used as probes. Probes were labeled with $\left[{ }^{32} \mathrm{P}\right] \mathrm{dCTP}$ using a DNA Labeling Kit ver. 2 (TaKaRa, Japan), and membranes were hybridized with ${ }^{32} \mathrm{P}$-labeled fragments at $65^{\circ} \mathrm{C}$ overnight. The membranes were washed 3 times with $1 \times \mathrm{SSC}, 1 \% \mathrm{SDS}$ for $3 \mathrm{~min}$ at room temperature; then once with $1 \times \mathrm{SSC}, 1 \% \mathrm{SDS}$ for $15 \mathrm{~min}$ at room temperature; then twice with $0.1 \times \mathrm{SSC}, 0.1 \% \mathrm{SDS}$ for $15 \mathrm{~min}$ at $65^{\circ} \mathrm{C}$.

\section{Semiquantitative or quantitative RT-PCR}

For cDNA synthesis, $1 \mu \mathrm{g}$ of total RNA was first treated with DNaseI (Sigma-Aldrich, USA) for $15 \mathrm{~min}$ at room temperature, and the enzyme was inactivated by heating at $70^{\circ} \mathrm{C}$ for $10 \mathrm{~min}$. Reverse transcription was performed with the ThermoScript RT-PCR system (Invitrogen, USA) according to the manufacturer's instructions. Synthesized cDNAs were purified using the Gel Extraction kit. Semiquantitative RT-PCR analysis for ThSOS1 and AtSOS1 expression was performed using $1 \mu \mathrm{l}$ of the cDNA, primer sets (ThSOS1; Exon13-14 forward 5'CCGAGACAGGAACAATGTTTAT-3' and Exon15-16 reverse 5'-AGTAAGCTGCCTGAACACCAT-3', AtSOS1; Exon13-14 forward 5'-AGGAGACTGGAACATTG TTTCT-3' and Exon15-16 reverse 5'-AGTAAGTTGCT TGCACACCATT-3') and BioTaq polymerase with the supplied buffer and dNTP (BIOLINE, UK). The PCR conditions were as follows: 30 cycles of $95^{\circ} \mathrm{C}$ for $30 \mathrm{~s}, 55^{\circ}$ $\mathrm{C}$ for $30 \mathrm{~s}$ and $72^{\circ} \mathrm{C}$ for $30 \mathrm{~s}$. A $10 \mu \mathrm{l}$ aliquot of each PCR reaction was separated on an agarose gel. The comparative expression analysis of ThSOS1 and ThSOS1S was performed by quantitative PCR with LightCycler Systems for Real-Time PCR (Roche Applied Science, Japan) using the LightCycler-FastStart DNA Master SYBR Green I kit (Roche Applied Science, Japan) and the primer sets 
(ThSOS1; Exon14-15 forward 5'- CGGTTTACCAGCCTCAAAGATACGAA-3' and reverse 5'- AAACGCTTGTAAGGCCTTATTCAGCAT-3', ThSOS1S; Exon14-15 forward 5'- TTTACCAGCCTCAAAGGGAAACTGTG3' and ThSOS1S specific reverse 5'- CACCTAACTA TGCCCGCTCAAGGA-3' and Actin2; forward 5'AGTGGTCGTACAACCGGTATTGT-3' and reverse 5'- GATGGCA TGAGGAAGAGAGAAAC-3') according to the manufacturer's instructions. The PCR conditions were as follows: 40 cycles of $95^{\circ} \mathrm{C}$ for $10 \mathrm{~s}, 55^{\circ} \mathrm{C}$ for $10 \mathrm{~s}$ and $72^{\circ} \mathrm{C}$ for $10 \mathrm{~s}$. The relative expressions were calculated using the Second Derivative Maximum Method on LightCycler Data Analysis software (Roche Applied Science, Japan). The Actin2 (At3g18730) coorthologous gene was used to normalize ThSOS1 and ThSOS1S expressions.

\section{Measurement of plant $\mathrm{Na}^{+}$content}

Two-week-old Arabidopsis and Thellungiella plants grown on $1 / 2 \mathrm{MS}$ agar plates were transferred to plates containing $1 / 2 \mathrm{MS}$ agar medium plus $250 \mathrm{mM} \mathrm{NaCl}$. Plants were harvested at $1,3,5,7,10,14,21$, and 28 days after transfer. For each sample, five plants were pooled and soaked in $5 \mathrm{~mL}$ sterile distilled water. The leaf-water mixture was boiled for $15 \mathrm{~min}$, filtered through a $0.2-\mu \mathrm{m}$ filter (Toyo Roshi Kaisha, Ltd.), and diluted 20-fold. The solution was analyzed by using a Shim-pack IC-C3/C3 (S) column (Shimadzu, Japan) on a Shimadzu PIA-1000 Personal Ion Analyzer (Shimadzu, Japan).

\section{Additional material}

Additional file 1: Table S1, Title. Predicted protein coding features, annotation for RIKEN T. halophila full-length cDNA (RTFL) collection. Abbreviations: AA, amino acid, CDS, coding sequence, NA, nucleic acid.

Additional file 2: Table S2, Title. Predicted 5', 3'-untranslated region of RIKEN T. halophila full-length cDNA (RTFL) collection. Abbreviation: UTR, untranslated region.

Additional file 3: Figure S1, Title. Arrangement of motifs identified in 5'-UTR regions of 30 orthologous gene pairs in Thellungiella and Arabidopsis.

Additional file 4: Table S3, Title. Motif sequences.

\begin{abstract}
Acknowledgements
We are grateful for the technical support provided by Kousuke Sugahara and Tatsuya Murata of the Faculty of Applied Bioscience, Tokyo University of Agriculture. The Arabidopsis accessions used in this study are maintained and provided by the RIKEN BRC through the National Bio-Resource Project of the MEXT, Japan. Characterization of full-length sequence of RTFL clones was supported by the National Bio-Resource Project of the MEXT, Japan. This work was supported by a Grant-in-aid for Young Scientists from the Ministry of Education, Culture, Sports, Science and Technology of Japan (T. T.), and by the Advanced Research Project of Tokyo University of Agriculture.
\end{abstract}

\section{Author details}

${ }^{1}$ Faculty of Applied Bioscience, Tokyo University of Agriculture, 1-1-1 Sakuragaoka, Setagaya-ku, Tokyo 156-8502, Japan. ${ }^{2}$ Kihara Institute for
Biological Research, Yokohama City University, Maioka 641-12, Totsuka, Yokohama 244-0813, Japan. ${ }^{3}$ Experimental Plant Division, BioResource Center, RIKEN Tsukuba Institute, 3-1-1 Koyadai, Tsukuba, Ibaraki 305-0074, Japan. ${ }^{4}$ RIKEN Genomic Sciences Center,1-7-22 Suehiro-cho, Tsurumi-ku, Yokohama, 230-0045, Japan. ${ }^{5}$ Current address: Comparative Genomics Laboratory, National Institute of Genetics, Yata 1111, Mishima, Shizuoka 4118540, JAPAN. ${ }^{6}$ RIKEN Plant Science Center, 1-7-22 Suehiro-cho, Tsurumi-ku, Yokohama, Kanagawa 230-0045, Japan.

\section{Authors' contributions}

$\Pi T$ contributed to and participated in the entire study and drafted the manuscript. KK performed the bioinformatics analyses (annotation, prediction of CDS, 5'-UTR and 3'-UTR and comparative analysis). TK carried out molecular biology studies and a part of bioinformatics analyses (comparative analysis). KY carried out the Northern analysis. AT conducted sequencing of the CDNA clones and registration in DDBJ. KM planned the study and obtained funding for the research. YS and ST helped draft the manuscript. KS and MS coordinated the project and helped draft the manuscript.

Received: 30 July 2010 Accepted: 24 November 2010 Published: 24 November 2010

\section{References}

1. Bressan RA, Zhang C, Zhang H, Hasegawa PM, Bohnert HJ, Zhu JK: Learning from the Arabidopsis experience. The next gene search paradigm. Plant Physiol 2001, 127(4):1354-1360.

2. Inan G, Zhang Q, Li P, Wang Z, Cao Z, Zhang H, Zhang C, Quist TM, Goodwin SM, Zhu J, Shi H, Damsz B, Charbaji T, Gong Q, Ma S, Fredricksen M, Galbraith DW, Jenks MA, Rhodes D, Hasegawa PM, Bohnert HJ, Joly RJ, Bressan RA, Zhu JK: Salt cress. A halophyte and cryophyte Arabidopsis relative model system and its applicability to molecular genetic analyses of growth and development of extremophiles. Plant Physiol 2004, 135(3):1718-1737.

3. Taji T, Seki M, Satou M, Sakurai T, Kobayashi M, Ishiyama K, Narusaka Y, Narusaka M, Zhu JK, Shinozaki K: Comparative genomics in salt tolerance between Arabidopsis and Arabidopsis-related halophyte salt cress using Arabidopsis microarray. Plant Physiol 2004, 135(3):1697-1709.

4. Volkov V, Amtmann A: Thellungiella halophila, a salt-tolerant relative of Arabidopsis thaliana, has specific root ion-channel features supporting $\mathrm{K}$ +/Na+ homeostasis under salinity stress. Plant J 2006, 48(3):342-353.

5. Gong Q, Li P, Ma S, Indu Rupassara S, Bohnert HJ: Salinity stress adaptation competence in the extremophile Thellungiella halophila in comparison with its relative Arabidopsis thaliana. Plant J 2005, 44(5):826-839.

6. Vera-Estrella R, Barkla BJ, Garcia-Ramirez L, Pantoja O: Salt stress in Thellungiella halophila activates $\mathrm{Na}+$ transport mechanisms required for salinity tolerance. Plant Physiol 2005, 139(3):1507-1517.

7. Kant S, Kant P, Raveh E, Barak S: Evidence that differential gene expression between the halophyte, Thellungiella halophila, and Arabidopsis thaliana is responsible for higher levels of the compatible osmolyte proline and tight control of $\mathrm{Na}+$ uptake in $\mathrm{T}$. halophila. Plant Cell Environ 2006, 29(7):1220-1234.

8. Li WY, Wong FL, Tsai SN, Phang TH, Shao G, Lam HM: Tonoplast-located $\mathrm{GmCLC1}$ and $\mathrm{GmNHX} 1$ from soybean enhance $\mathrm{NaCl}$ tolerance in transgenic bright yellow (BY)-2 cells. Plant Cell Environ 2006, 29(6):1122-1137.

9. Wong CE, Li Y, Whitty BR, Diaz-Camino C, Akhter SR, Brandle JE, Golding GB, Weretilnyk EA, Moffatt BA, Griffith M: Expressed sequence tags from the Yukon ecotype of Thellungiella reveal that gene expression in response to cold, drought and salinity shows little overlap. Plant $\mathrm{Mol}$ Biol 2005, 58(4):561-574.

10. Wong CE, Li Y, Labbe A, Guevara D, Nuin P, Whitty B, Diaz C, Golding GB, Gray GR, Weretilnyk EA, Griffith M, Moffatt BA: Transcriptional profiling implicates novel interactions between abiotic stress and hormonal responses in Thellungiella, a close relative of Arabidopsis. Plant Physiol 2006, 140(4):1437-1450

11. Zhu JK: Plant salt tolerance. Trends Plant Sci 2001, 6(2):66-71.

12. Irish VF, Benfey PN: Beyond Arabidopsis. Translational biology meets evolutionary developmental biology. Plant Physiol 2004, 135(2):611-614. 
13. Alexandrov NN, Troukhan ME, Brover W, Tatarinova T, Flavell RB, Feldmann KA: Features of Arabidopsis genes and genome discovered using full-length cDNAs. Plant Mol Biol 2006, 60(1):69-85.

14. Castelli V, Aury JM, Jaillon O, Wincker P, Clepet C, Menard M, Cruaud C, Quetier F, Scarpelli C, Schachter V, Temple G, Caboche M, Weissenbach J, Salanoubat M: Whole genome sequence comparisons and "full-length" cDNA sequences: a combined approach to evaluate and improve Arabidopsis genome annotation. Genome Res 2004, 14(3):406-413.

15. Haas BJ, Delcher AL, Mount SM, Wortman JR, Smith RK, Hannick LI, Maiti R, Ronning CM, Rusch DB, Town CD, Salzberg SL, White O: Improving the Arabidopsis genome annotation using maximal transcript alignment assemblies. Nucleic Acids Res 2003, 31(19):5654-5666.

16. Taji T, Sakurai T, Mochida K, Ishiwata A, Kurotani A, Totoki Y, Toyoda A, Sakaki Y, Seki M, Ono H, Sakata Y, Tanaka S, Shinozaki K: Large-scale collection and annotation of full-length enriched CDNAs from a model halophyte, Thellungiella halophila. BMC Plant Biol 2008, 8:115.

17. Chinnusamy V, Schumaker K, Zhu JK: Molecular genetic perspectives on cross-talk and specificity in abiotic stress signalling in plants. J Exp Bot 2004, 55(395):225-236.

18. Shi H, Lee BH, Wu SJ, Zhu JK: Overexpression of a plasma membrane Na $+/ \mathrm{H}+$ antiporter gene improves salt tolerance in Arabidopsis thaliana. Nat Biotechnol 2003, 21(1):81-85.

19. Batelli G, Verslues PE, Agius F, Qiu Q, Fujii H, Pan S, Schumaker KS, Grillo S, Zhu JK: SOS2 promotes salt tolerance in part by interacting with the vacuolar $\mathrm{H}+$-ATPase and upregulating its transport activity. Mol Cell Biol 2007, 27(22):7781-7790.

20. Shi H, Ishitani M, Kim C, Zhu JK: The Arabidopsis thaliana salt tolerance gene SOS1 encodes a putative $\mathrm{Na}+/ \mathrm{H}+$ antiporter. Proc Natl Acad Sci USA 2000, 97(12):6896-6901.

21. Oh DH, Leidi E, Zhang Q, Hwang SM, Li Y, Quintero FJ, Jiang X, D'Urzo MP, Lee SY, Zhao Y, Bahk JD, Bressan RA, Yun DJ, Pardo JM, Bohnert HJ: Loss of halophytism by interference with SOS1 expression. Plant Physiol 2009, 151(1):210-222.

22. Nah G, Pagliarulo CL, Mohr PG, Luo M, Sisneros N, Yu Y, Collura K, Currie J, Goicoechea JL, Wing RA, Schumaker KS: Comparative sequence analysis of the SALT OVERLY SENSITIVE1 orthologous region in Thellungiella halophila and Arabidopsis thaliana. Genomics 2009, 94(3):196-203.

23. Jia J, Fu J, Zheng J, Zhou X, Huai J, Wang J, Wang M, Zhang Y, Chen $X$, Zhang J, Zhao J, Su Z, Lv Y, Wang G: Annotation and expression profile analysis of 2073 full-length cDNAs from stress-induced maize (Zea mays L.) seedlings. Plant $J$ 2006, 48(5):710-727.

24. Kikuchi S, Satoh K, Nagata T, Kawagashira N, Doi K, Kishimoto N, Yazaki J, Ishikawa M, Yamada H, Ooka H, Hotta I, Kojima K, Namiki T, Ohneda E, Yahagi W, Suzuki K, Li CJ, Ohtsuki K, Shishiki T, Otomo Y, Murakami K, lida Y, Sugano S, Fujimura T, Suzuki Y, Tsunoda Y, Kurosaki T, Kodama T, Masuda H, Kobayashi M, Xie Q, Lu M, Narikawa R, Sugiyama A, Mizuno K, Yokomizo S, Niikura J, Ikeda R, Ishibiki J, Kawamata M, Yoshimura A, Miura J, Kusumegi T, Oka M, Ryu R, Ueda M, Matsubara K, Kawai J, Carninci P, Adachi J, Aizawa K, Arakawa T, Fukuda S, Hara A, Hashizume W, Hayatsu N, Imotani K, Ishii Y, Itoh M, Kagawa I, Kondo S, Konno H, Miyazaki A, Osato N, Ota Y, Saito R, Sasaki D, Sato K, Shibata K, Shinagawa A, Shiraki T, Yoshino M, Hayashizaki Y, Yasunishi A: Collection, mapping, and annotation of over 28,000 cDNA clones from japonica rice. Science 2003, 301(5631):376-379

25. Ralph SG, Chun HJ, Cooper D, Kirkpatrick R, Kolosova N, Gunter L, Tuskan GA, Douglas CJ, Holt RA, Jones SJ, Marra MA, Bohlmann J: Analysis of 4,664 high-quality sequence-finished poplar full-length cDNA clones and their utility for the discovery of genes responding to insect feeding. BMC Genomics 2008, 9:57.

26. Huang X, Madan A: CAP3: A DNA sequence assembly program. Genome Res 1999, 9(9):868-877.

27. Christoffels A, Bartfai R, Srinivasan H, Komen H, Orban L: Comparative genomics in cyprinids: common carp ESTs help the annotation of the zebrafish genome. BMC Bioinformatics 2006, 7(Suppl 5):S2.

28. Counillon $\mathrm{L}$, Pouyssegur J: The expanding family of eucaryotic $\mathrm{Na}(+) / \mathrm{H}(+)$ exchangers. J Biol Chem 2000, 275(1):1-4.

29. Putney LK, Denker SP, Barber DL: The changing face of the $\mathrm{Na}+/ \mathrm{H}+$ exchanger, NHE1: structure, regulation, and cellular actions. Annu Rev Pharmacol Toxicol 2002, 42:527-552.

30. Hamada A, Hibino T, Nakamura T, Takabe $\mathrm{T}: \mathrm{Na}+/ \mathrm{H}+$ antiporter from Synechocystis species PCC 6803, homologous to SOS1, contains an aspartic residue and long C-terminal tail important for the carrier activity. Plant Physiol 2001, 125(1):437-446.

31. Katiyar-Agarwal S, Zhu J, Kim K, Agarwal M, Fu X, Huang A, Zhu JK: The plasma membrane $\mathrm{Na}+/ \mathrm{H}+$ antiporter SOS1 interacts with $\mathrm{RCD} 1$ and functions in oxidative stress tolerance in Arabidopsis. Proc Natl Acad Sci USA 2006, 103(49):18816-18821.

32. Utsugi J, Inaba K, Kuroda T, Tsuda M, Tsuchiya T: Cloning and sequencing of a novel $\mathrm{Na}+/ \mathrm{H}+$ antiporter gene from Pseudomonas aeruginosa. Biochim Biophys Acta 1998, 1398(3):330-334.

33. Zhu JK, Liu J, Xiong L: Genetic analysis of salt tolerance in arabidopsis. Evidence for a critical role of potassium nutrition. Plant Cell 1998, 10(7):1181-1191

34. Apse MP, Sottosanto JB, Blumwald E: Vacuolar cation/H+ exchange, ion homeostasis, and leaf development are altered in a T-DNA insertional mutant of AtNHX1, the Arabidopsis vacuolar $\mathrm{Na}+/ \mathrm{H}+$ antiporter. Plant $J$ 2003, 36(2):229-239.

35. Horie T, Motoda J, Kubo M, Yang H, Yoda K, Horie R, Chan WY, Leung HY, Hattori K, Konomi M, Osumi M, Yamagami M, Schroeder Jl, Uozumi N: Enhanced salt tolerance mediated by AtHKT1 transporter-induced Na unloading from xylem vessels to xylem parenchyma cells. Plant J 2005, 44(6):928-938.

36. Apse MP, Aharon GS, Snedden WA, Blumwald E: Salt tolerance conferred by overexpression of a vacuolar $\mathrm{Na}+/ \mathrm{H}+$ antiport in Arabidopsis. Science 1999, 285(5431):1256-1258.

37. Rus A, Lee BH, Munoz-Mayor A, Sharkhuu A, Miura K, Zhu JK, Bressan RA, Hasegawa PM: AtHKT1 facilitates $\mathrm{Na}+$ homeostasis and $\mathrm{K}+$ nutrition in planta. Plant Physiol 2004, 136(1):2500-2511.

38. Rubio F, Schwarz M, Gassmann W, Schroeder Jl: Genetic selection of mutations in the high affinity $\mathrm{K}+$ transporter HKT1 that define functions of a loop site for reduced $\mathrm{Na}+$ permeability and increased $\mathrm{Na}+$ tolerance. J Biol Chem 1999, 274(11):6839-6847.

39. Maser P, Hosoo Y, Goshima S, Horie T, Eckelman B, Yamada K, Yoshida K, Bakker EP, Shinmyo A, Oiki S, Schroeder I, Uozumi N: Glycine residues in potassium channel-like selectivity filters determine potassium selectivity in four-loop-per-subunit HKT transporters from plants. Proc Natl Acad Sci USA 2002, 99(9):6428-6433.

40. Rice P, Longden I, Bleasby A: EMBOSS: the European Molecular Biology Open Software Suite. Trends Genet 2000, 16(6):276-277.

41. Altschul SF, Madden TL, Schaffer AA, Zhang J, Zhang Z, Miller W, Lipman DJ: Gapped BLAST and PSI-BLAST: a new generation of protein database search programs. Nucleic Acids Res 1997, 25(17):3389-3402.

42. Kawai J, Shinagawa A, Shibata K, Yoshino M, Itoh M, Ishii Y, Arakawa $T$, Hara A, Fukunishi Y, Konno H, Adachi J, Fukuda S, Aizawa K, Izawa M, Nishi K, Kiyosawa H, Kondo S, Yamanaka I, Saito T, Okazaki Y, Gojobori T, Bono H, Kasukawa T, Saito R, Kadota K, Matsuda H, Ashburner M, Batalov S, Casavant T, Fleischmann W, Gaasterland T, Gissi C, King B, Kochiwa H, Kuehl P, Lewis S, Matsuo Y, Nikaido I, Pesole G, Quackenbush J, Schriml LM, Staubli F, Suzuki R, Tomita M, Wagner L, Washio T, Sakai K, Okido T, Furuno M, Aono H, Baldarelli R, Barsh G, Blake J, Boffelli D, Bojunga N, Carninci P, de Bonaldo MF, Brownstein MJ, Bult C, Fletcher C, Fujita M, Gariboldi M, Gustincich S, Hill D, Hofmann M, Hume DA, Kamiya M, Lee NH, Lyons P, Marchionni L, Mashima J, Mazzarelli J, Mombaerts P, Nordone P, Ring B, Ringwald M, Rodriguez I, Sakamoto N, Sasaki H, Sato K, Schonbach C, Seya T, Shibata Y, Storch KF, Suzuki H, Toyo-oka K, Wang KH, Weitz C, Whittaker C, Wilming L, Wynshaw-Boris A, Yoshida K, Hasegawa Y, Kawaji H, Kohtsuki S, Hayashizaki Y: Functional annotation of a full-length mouse cDNA collection. Nature 2001, 409(6821):685-690.

doi:10.1186/1471-2229-10-261

Cite this article as: Taji et al:: Comparative genomic analysis of 1047 completely sequenced cDNAs from an Arabidopsis-related model halophyte, Thellungiella halophila. BMC Plant Biology 2010 10:261. 